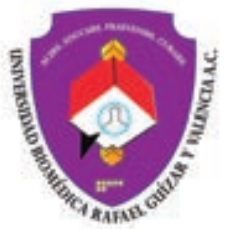

Vol. 8, Núm. 1

Enero-Junio 2021. pp. 7-12

\title{
Evaluación de la agudeza visual en niños de la Escuela Primaria «Úrsulo Galván», Turno Matutino de Xalapa, Veracruz
}

\author{
Evaluation of visual acuity in children of the «Úrsulo Galván», \\ Elementary School, morning shift Xalapa, Veracruz \\ Gerardo José Roberto Ortega Matías, * \\ Karla Graciela Hernández Hernández, ${ }^{\ddagger}$ Francisco Aguilar Rebolledo ${ }^{\S}$
}

\section{RESUMEN}

Introducción: Los déficits en visión actualmente traen consigo diversos problemas de gran importancia para el desarrollo neuronal y aprendizaje. Objetivo: Analizar la agudeza visual de niños escolares de la Escuela Primaria «Úrsulo Galván». Material y métodos: Se realizó una evaluación a los niños con edades de entre los cinco y los 11 años de edad, quienes se encontraban en los grupos de primero a sexto grado. El universo estuvo constituido por un total de 348 niños, con una prevalencia del sexo femenino de 185 niñas (53\%) y 163 niños (47\%). La evaluación se realizó en 696 ojos a los cuales se les tomó la agudeza visual con cartilla de Snellen para quienes sabían leer adecuadamente y cartilla de figuras para niños de menor edad que no conocían aún las letras en su totalidad. Se distribuyeron en grupos de un promedio de 25 niños, los ojos se distribuyeron por rango de agudeza visual (AV). Entre otras variables optométricas que se tomaron en cuenta, la más importante es la AV, para la cual se consideran como valores normales 20/20 en notación Snellen (1.00 decimal, 1 MAR y 1/1 en fracción Snellen), se consideran valores superiores a lo normal mayores a 20/20 (20/15, 1.33 decimal, 0.45 MAR y $1 / .75$ en fracción Snellen), déficit leve valores que van de 20/25 a 20/40 en notación Snellen, déficit moderado con valores de

\section{ABSTRACT}

Introduction: Vision deficits currently bring with them various problems, of great importance for their neural development and learning. Objective: To analyze the visual acuity of elementary school children «Úrsulo Galván». Material and methods: An evaluation was carried out on children with ages between 5 to 11 years old, who were in the groups from 1st to 6th grade. The universe consisted of a total of 348 boys, with a prevalence in the female gender with 185 girls (53\%) and 163 boys (47\%) of the male gender. The evaluation was carried out in 696 eyes in which visual acuity was taken with a Snellen chart for those who knew how to read adequately and a chart of figures for younger children who did not yet know the letters in their entirety, they were distributed in groups of one average of 25 children, the eyes were distributed by range of Visual Acuity (VA). Among other optometric variables that were taken into account is the VA, for which 20/20 are considered normal values in Snellen notation (1.00 decimal, 1 MAR and 1/1 in Snellen fraction) are considered values higher than normal greater than 20/20 (20/15, 1.33 decimal, $0.45 \mathrm{MAR}$, and $1 / .75$ in Snellen fraction), mild deficit values ranging from 20/25 to 20/40 in Snellen notation, moderate deficit values from 20/50 to $20 / 80$ and severe deficit values of 20/100 or more.

* Licenciado en Optometría por el Instituto Politécnico Nacional. Coordinador de la Licenciatura en Optometría y Rehabilitación Visual en la Universidad Biomédica «Rafael Guízar y Valencia».

‡ Licenciado en Optometría por el Instituto Politécnico Nacional.

§ Rector e Investigador de la Universidad Biomédica «Rafael Guízar y Valencia».

$$
\text { Correspondencia: }
$$

Lic. Opt. Gerardo José Roberto Ortega Matías

Coordinador de la Licenciatura en Optometría y Rehabilitación Visual de la Universidad Biomédica «Rafael Guízar y Valencia».

E-mail: optometria@universidadbiomedicargyv.com

Recibido: 01-04-2021. Aceptado: 16-05-2021.

Citar como: Ortega MGJR, Hernández HKG, Aguilar RF. Evaluación de la agudeza visual en niños de la Escuela Primaria «Úrsulo Galván», Turno Matutino de Xalapa, Veracruz. Plast Restaur Neurol. 2021;8 (1): 7-12. https://dx.doi.org/10.35366/101199 
20/50 a 20/80 y déficit severo con valores de 20/100 o más. Resultados: De los 696 ojos, 312 (44.82\%) tienen una AV de 20/20 o superior (20/20,32.04\%) corresponden a 223 ojos y 20/15 a 89 ojos (12.78\%) y 55.18\% tiene una AV menor a 20/20 que corresponde a una AV leve, moderada o severa. Conclusiones: En México los estudios que abordan este problema son escasos al igual que en Latinoamérica. Los valores de la agudeza visual (AV) se basan en estándares norteamericanos o europeos. Son pocos los casos en los que la AV es menor a 20/100; sin embargo, existen, y para esta población este tipo de AV se considera un factor de riesgo bastante alto y de peligro, más aún si no son diagnosticados a tiempo por el especialista, en su mayoría el problema puede pasar desapercibido y no ser detectado hasta la edad adulta.

Palabras clave: Agudeza visual, corrección visual, detección, corrección.
Results: Of the 696 eyes, 312 eyes (44.82\%) have a VA of 20/20 or higher (20/20 32.04\% corresponding to 223 eyes and 20/15 89 eyes (12.78\%) and $55.18 \%$ have a lower VA to $20 / 20$ corresponding to a mild, moderate, or severe VA. Conclusions: In Mexico, studies that address this problem are scarce, as in Latin America. On this subject, and also in Latin America, the visual acuity (VA) values are based on North American or European standards. There are few cases where the VA is less than 20/100, however there are and for this population and age this type of VA is considered a fairly high risk factor and danger, even more so if they are not diagnosed in time by the specialist. That in its majority being only one eye of two that a normal subject has, it can go unnoticed and not be detected until adulthood.

Keywords: Visual acuity, visual correction, detection, correction.

\section{INTRODUCCIÓN}

Se conoce que $80 \%$ de la información proveniente del mundo exterior pasa a través del sistema visual; debido a tal porcentaje es la importancia de una buena visión y detección oportuna de las anomalías refractivas para evitar ambliopías de todo tipo, tropias, anomalías binoculares y/o de percepción. Las alteraciones visuales generan consecuencias adversas en el ser humano, pero especialmente en los niños, ya que se encuentran en el periodo de neurodesarrollo, lo cual es un factor importante que puede repercutir en el rendimiento escolar. ${ }^{1,2}$

De acuerdo con la Organización Mundial de la Salud (OMS) recientemente se emitió un documento para las Américas, el cual estima que 7.3 millones de niños presentan deficiencia visual. Los estudios realizados en México destacan que alrededor de 30 a 40\% presentan deficiencias visuales que se pueden corregir. ${ }^{3}$ En Latinoamérica los estudios muestran alteraciones visuales que van desde 35 hasta $50 \%$ y repercuten en su rendimiento escolar. ${ }^{3,4}$

La Organización de las Naciones Unidas (ONU) hizo un boletín en 2019 en el cual se expone que la ceguera a nivel mundial es, en su mayoría, prevenible si la detección es oportuna. Al seguir esa línea, se debe entender que el licenciado en Optometría debe ser el encargado de esta detección, así como del correcto tratamiento o derivación a servicios especializados. Asimismo, al ser éste el especialista de primer contacto debería siempre estar al alcance de toda la población.

En México los estudios relacionados con la salud visual son escasos y aún es una deuda que está por cumplirse. Se sospecha que alrededor de $30 \%$ de los infantes tienen defectos visuales que pudieran influir en la maduración y el desarrollo cerebral, ${ }^{5}$ lo cual dificulta la red neuronal para la adquisición de otras habilidades enhebradas dentro del proceso de la maduración cerebral, como son la capacidad motora, el equilibrio, la orientación visoespacial, el lenguaje y la lectoescritura (algunas alteraciones de estas habilidades se atribuyen a los problemas de digrafía y disartria) y otras capacidades cognitivas necesarias para un aprendizaje significativo y otras capacidades de síntesis, praxis, orientación y juicio $^{6}$ (independientemente de los trastornos en el aprovechamiento escolar y el aprendizaje).

Nuestro objetivo en este trabajo es estudiar la agudeza visual en niños en quienes el proceso de maduración y desarrollo relacionado con la adquisición de habilidades motoras y cognitivas requieren fundamentalmente de la recepción de estímulos visuales normales traducidos en una buena agudeza visual.

\section{MATERIAL Y MÉTODOS}

Se trata de un estudio observacional, descriptivo y transversal. Se realizó la evaluación en niños de la Escuela Primaria «Úrsulo Galván». La edad correspondió de los cinco a los 11 años de edad, quienes se encontraban en los grupos de primero a sexto grado. El universo estuvo constituido por un total de 348 niños, con una prevalencia del sexo femenino de 185 niñas (53\%) y 163 niños (47\%). Para un correcto análisis de la información se tomó en cuenta cada ojo de cada individuo, por lo que se tomó en cuenta un total de 696 ojos.

Para la evaluación de la agudeza visual (AV) se utilizó una cartilla de tipo iletrados (figuras) para niños de cinco y seis años, en el cual se toma la AV de cada ojo como buena referencia si el niño dice bien cuatro de cinco figuras por línea; en el caso de los niños mayores de siete años en adelante se utilizó una cartilla Snellen de letras siguiendo los mismos lineamientos, si el niño dice bien cuatro de cinco 
letras, se toma como buena. La distancia que se ocupó fue de tres metros, en la cual se hizo la conversión del tamaño de letras.

Se dividieron en grupos de un promedio de 25 niños y los ojos se distribuyeron por rango de agudeza visual (AV). Entre otras variables optométricas que se tomaron en cuenta, la más importante es la $\mathrm{AV}$, para la cual se consideran como valores normales 20/20 en notación Snellen (1.00 decimal, 1 MAR y $1 / 1$ en fracción Snellen), se consideran valores superiores a lo normal mayores a 20/20 (20/15, 1.33 decimal, 0.45 MAR y $1 / .75$ en fracción Snellen), déficit leve valores que van de 20/25

Tabla 1: Donde indica los valores de agudeza visual en fracción Snellen.

\begin{tabular}{cc} 
& Agudeza visual (déficit) \\
\hline $20 / 20$ & Normal \\
$20 / 25$ a $20 / 40$ & Leve \\
$20 / 50$ a $20 / 80$ & Moderado \\
$20 / 100$ o más & Severo \\
\hline
\end{tabular}

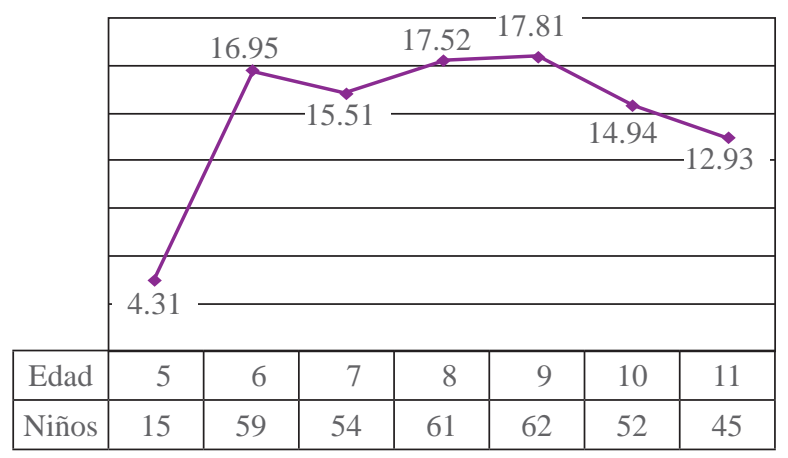

Figura 1: Se describe la distribución por edades de la población. a 20/40 en notación Snellen, déficit moderado con valores de 20/50 a 20/80 y déficit severo con valores de 20/100 o más (Tabla 1).

\section{PROCEDIMIENTO}

Se le explica al niño que tape con un oclusor su ojo izquierdo para comenzar la evaluación con el ojo derecho, se le pide que indique las letras o figuras que alcanza a leer iniciando con la más grande y hasta la más pequeña, en todo momento el evaluador observa al niño. Se repite el procedimiento con el ojo contrario y se registra el valor individual para cada ojo.

\section{RESULTADOS}

El sexo correspondió a 185 niñas (53\%) y 163 niños (47\%). Dentro del total de la población $4.34 \%$ fueron infantes de cinco años de edad ( $n=15) ; 16.95 \%$ correspondieron al grupo de seis años $(n=59) ; 15.51 \%$ tuvieron siete años $(n=54) ; 17.52 \%$ fueron de ocho años $(n=61) ; 17.81 \%$ correspondieron al grupo de nueve años $(n=62)$, siendo la mayor prevalencia esta edad; $14.94 \%$ tuvieron 10 años $(n=52)$ y, por último, $12.93 \%$ fueron niños de 11 años $(n=45)$ (Figura 1).

La edad de los niños corresponde a la matrícula de una escuela pública normal dentro de nuestro país, es decir, los datos obtenidos se pueden extrapolar a otras entidades de la República Mexicana (Figura 1).

Al tener en cuenta que nuestra mayor prevalencia es en edades de entre los ocho y los nueve años, y que además se encontraron datos relevantes en la agudeza visual, es importante entender que dentro del desarrollo visual los primeros tres años son de gran relevancia, posteriormente hasta los siete años es cuando el desarrollo de las estructuras visuales

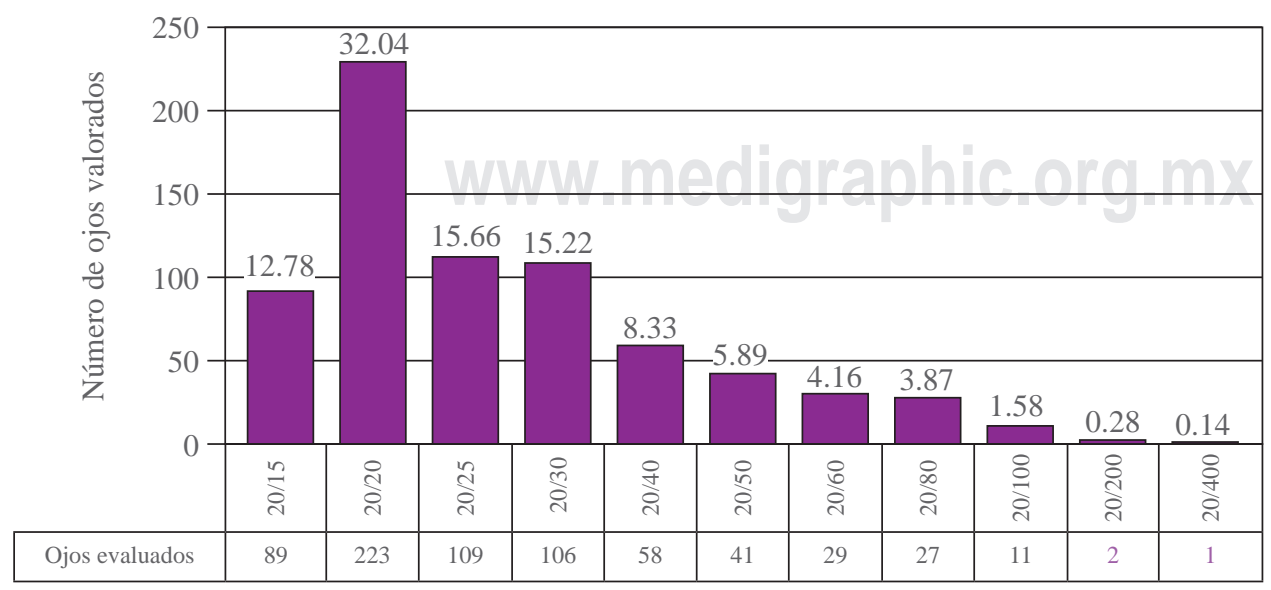

Figura 2:

Se observa una prevalencia alta de una agudeza visual normal, seguida de una agudeza visual leve, moderada o severa. 
Tabla 2: Observamos los porcentajes en relación al grado de agudeza visual.

\begin{tabular}{cr}
\hline Déficit & $\%$ \\
\hline $20 / 20$ o menos & 45 \\
Leve & 39 \\
Moderado & 14 \\
Severo & 2 \\
\hline
\end{tabular}

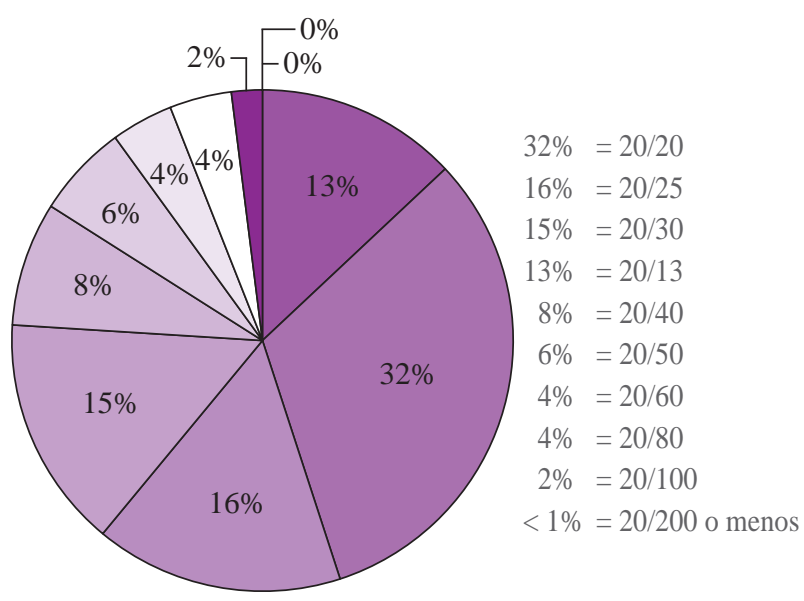

Figura 3: Porcentajes por prevalencia de déficit de agudeza visual.

culmina, lo cual también es elemental para el desarrollo neurológico y visual.

Al momento de realizar la evaluación se decidió que se debe evaluar cada ojo para determinar el estado de la $\mathrm{AV}$ y de esta forma tener una muestra más precisa. Es entonces que se evaluaron un total de 696 ojos, de los cuales se explica la prevalencia en la Figura 2.

Se determinó el estado de la AV por población, siendo un dato muy interesante, ya que en proporción $44.82 \%$ tiene una AV de 20/20 o superior (20/20, $32.04 \%$ correspondiendo a 223 ojos y 20/15, 12.78\% correspondiendo a 89 ojos, respectivamente) y $55.18 \%$ tiene una AV menor a 20/20 correspondiendo a una AV leve, moderada o severa (Tabla 2).

Los resultados obtenidos por grupos de AV se muestran en la Figura 3.

Los grupos en los que se encontró el problema de la $\mathrm{AV}$ en algún grado correspondieron a los grupos etarios de seis y 11 años, se tomaron valores donde la población en su mayoría tiene una AV igual o peor a 20/25, con el entendido de que el sistema visual se termina de desarrollar y madurar posterior a los siete años y eso podría justificar la poca población de seis años con dicha normalidad en la AV (Figura 4). Sin embargo, no es así con el grupo de los 11 años, quienes ya han terminado su desarrollo. En la Figura 5 se observa la prevalencia de AV por edad de seis años.

Se encuentra que la prevalencia de AV es de 20/25 con un total de 41 niños (36.28\%) y de 20/20 para siete niños (6.19\%), donde se puede justificar que el desarroIlo del sistema visual no ha culminado y sin embargo, es alarmante encontrar casos en los que la AV es igual o menor a 20/50, en nuestro estudio se encontró un total de 26 ojos con este déficit (22.98\%). Cabe señalar que en estas situaciones el médico de primer contacto debe definir si la mala AV es causada por un error refractivo o por una patología de medios ópticos u otra condición que pudiera representar un peligro para la integridad o vida del paciente.

En lo que corresponde al grupo etario de los 11 años, el cual comprende a 90 niños, la prevalencia de una AV normal es de $17.77 \%$ (16 ojos) y el restante número

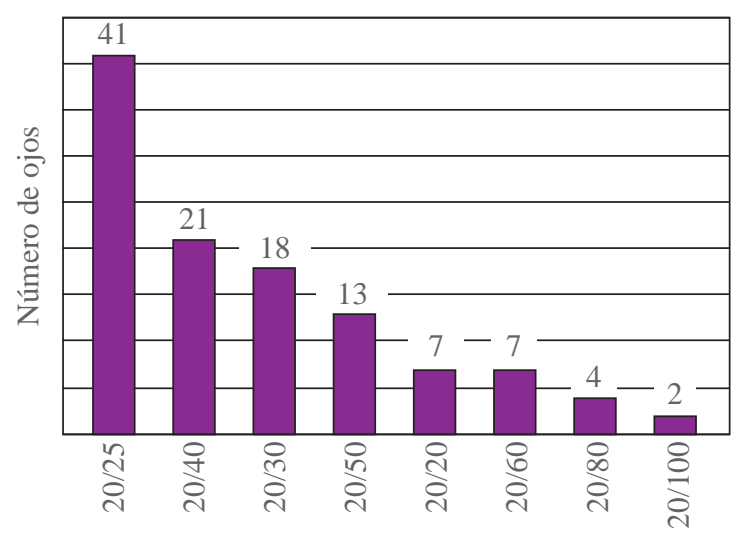

Figura 4: Resultados obtenidos en la población etaria de los seis años, destaca la alteración de la agudeza visual.

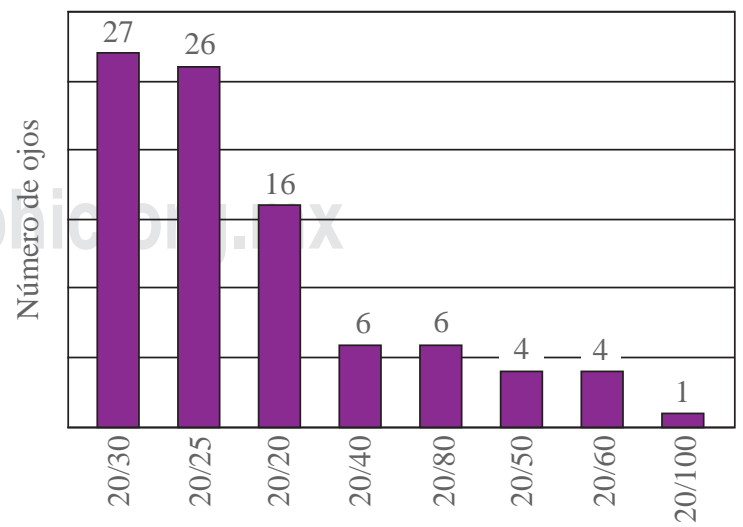

Figura 5: Resultados obtenidos en la población etaria de los 11 años, destaca la alteración de la agudeza visual. 
de ojos a una AV de 20/25 de igual o más, siendo el mayor número de ojos de los niños la AV de 20/30, la cual puede considerarse una pérdida de la AV leve.

En la población de los cinco años se halló que 15 ojos se encontraban con una AV de 20/20 (50\%); 10 ojos con déficit leve (33.33\%); cuatro ojos con déficit moderado (13.33\%) y un ojo con déficit severo (3.33\%).

El grupo de siete años siguió esta tendencia: 59 ojos se encontraban con una AV de 20/20 o mejor (54.62\%); 37 ojos con déficit leve (34.25\%); 12 con un déficit moderado $(11.1 \%)$ y no se encontró déficit severo de AV en esta población.

Con respecto a los resultados del grupo de edad correspondiente a los ocho, nueve y 10 años se encontró que 173 ojos se encontraban en valores normales de la AV (49.42\%); 109 ojos con un déficit leve (31.14\%); 58 ojos con un déficit moderado (16.57\%) y 10 ojos con un déficit severo (2.85\%).

\section{DISCUSIÓN}

Como lo menciona la Guía Práctica Clínica,7 "El examen ocular y la evaluación de la agudeza visual son fundamentales para la detección de alteraciones del sistema visual, con lo que sería factible identificar problemas estructurales y del desarrollo de la visión como los errores e la refracción, así como datos de alarma que indiquen otras enfermedades sistémicas".

Estos resultados discrepan un poco con lo encontrado por Capetillo BO y colaboradores, ${ }^{8}$ quienes exponen que cerca de $4.62 \%$ tiene una AV con un déficit leve en contraste con nuestro estudio que muestra un valor de $32 \%$.

Si estos defectos refractivos no se previenen adecuadamente, pueden influir en el neurodesarrollo de los niños; en consecuencia, el déficit de la visión tiene múltiples efectos en todo aspecto de la vida, sobre todo el aprendizaje. ${ }^{9-11}$ Por este motivo, las instituciones educativas deberán hacer un esfuerzo importante para apoyar el tamizaje visual de los niños para un adecuado desempeño dentro del aula. ${ }^{12}$ Esto refuerza lo realizado por Muñoz F y colaboradores, en el que los déficits astenópicos en la AV tienen una asociación altamente significativa entre el defecto de la AV y el rendimiento escolar $(p<0.006) .{ }^{13}$ Asimismo, la afectación principal fue a nivel de redes neuronales, donde la visión se integra a circuitos cerebrales del conocimiento y aprendizaje; sin embargo, no se encontró relación con dolor de cabeza o alteraciones locales como ojo rojo, lagrimeo o prurito. ${ }^{13}$

Por otro lado, son pocos los casos donde la AV es menor de 20/100; no obstante, existen, y para esta población este tipo de AV se considera un factor de riesgo para su integridad en cuanto al desarrollo cerebral y sus conexiones con otras habilidades en plena etapa del neurodesarrollo, más aún si no son diagnosticados a tiempo por el especialista, en su mayoría el problema puede pasar desapercibido y no ser detectado hasta la edad adulta. Por lo tanto, es importante examinar de manera individual y en conjunto la agudeza visual.

Si bien la prevalencia es mayor en AV de 20/20 o mejor, hay población que requiere algún tipo de corrección o su desarrollo visual no ha culminado del todo y hay que prestar mayor atención para poder determinar su estado refractivo certero con métodos objetivos de refracción y pruebas subjetivas, de esta forma se determina su estado refractivo y es probable que en su mayoría puedan corregirse, es decir, si se detecta un defecto leve en etapas tempranas, se hace la corrección y en el seguimiento, al llegar a una maduración cerebral mayor, se alcanza la normalidad de la AV.

En el grupo de edad de 11 años la alteración de la AV fue de $82.23 \%$, por lo que es importante determinar si es causada por un error refractivo o por alguna patología que no permita alcanzar la mejoría de la AV. Por ello se recomienda que en esta edad sea obligatoria la evaluación de la AV por un servicio de atención primaria de la visión para determinar el estado refractivo y posteriormente su derivación a otros servicios para diagnosticar el motivo de la alteración visual y establecer los mecanismos para su corrección oportuna.

El licenciado en Optometría es entonces el profesional de primer nivel quien diagnostica y da tratamiento a anomalías refractivas, acomodativas y de segmento anterior a nivel primario. Se debe entender la importancia de una buena visión en esta etapa, ya que es crítico contar con las herramientas necesarias para un adecuado desarrollo general y neuronal.

Además de estar preocupados, la Universidad Biomédica Rafael Guízar y Valencia y el Departamento de Investigación en visión, en cuanto a que todos los niños tengan la oportunidad de ser evaluados de su función visual para alcanzar el máximo potencial de su desarrollo cerebral a través de la visión.

La Universidad Biomédica Rafael Guízar y Valencia está comprometida con la población para la detección oportuna de enfermedades metabólicas como la diabetes mellitus, descontrol de grasas (conocidas como dislipidemia), entre otras, y se compromete a realizar trabajo de calidad, empatía, siendo nuestra base la ciencia y el humanismo. 


\section{CONCLUSIONES}

Se puede concluir que, si bien hay un mayor porcentaje de alumnos con una AV de 20/20 o mejor (44.82\%), también existe una gran cantidad (55.28\%) de población que requiere de una detección temprana en caso de presentar alguna anomalía refractiva o patológica que puede detectarse y tratarse en estadios tempranos para evitar trastornos en el aprendizaje y su neurodesarrollo en la plasticidad cerebral para la adquisición de otras habilidades relacionadas en las redes neuronales de la vía visual. Es importante recalcar que el grupo de edad que se observa con mayor vulnerabilidad es el grupo de seis y 11 años, ya que presentan grandes cantidades de déficits de visión, y en ellos habrá que poner mayor énfasis para determinar su estatus.

La mayoría de los estudios de Latinoamérica y algunos de México concluyen que los trastornos de la agudeza visual pueden ir desde 35 hasta $49.23 \%$, siendo un valor en el que se demuestra que ésta influye directamente en el desarrollo intelectual del escolar, lo que alienta a realizar un tamizaje visual cada año, especialmente en niños de primaria y secundaria.

El valor de nuestro estudio ratifica los esfuerzos previos de otros investigadores en ayudar con proyectos para determinar la agudeza visual de todos los niños posibles y corregir sus defectos para lograr un desarrollo cognitivo normal y un aprendizaje significativo.

Por tanto, es necesario que los optometristas realicen investigaciones y evaluaciones en todos los niños posibles y se unan al esfuerzo de la Universidad Biomédica Rafael Guízar y Valencia de una salud visual para todos.

Conflicto de intereses: No existe ningún conflicto de intereses, ni se recibió ningún apoyo económico de ningún laboratorio.

Agradecimientos: Todos los apoyos los brindó la Universidad Biomédica Rafael Guízar y Valencia.

\section{REFERENCIAS}

1. Muñoz F, Rebolleda G, Puerto B. Defectos de agudeza visual. An Pediatr Contin. 2006; [citado 01 Oct 2015];4(5):324-329. Disponible en: https://www.elsevier.es/index php?p=revista\&pRevista=pdfsim ple\& $\mathrm{pii}=S 1696281806736332 \& \mathrm{r}=51$
2. Verrone P, Simi M. Prevalencia de agudeza visual baja y trastornos oftalmológicos en niños de seis años de la ciudad de Santa Fe. Arch Argent Pediatr. 2008;[citado 01 Oct 2015]106(4):328333. Disponible en: https://www.sap.org.ar/docs/publicaciones/ archivosarg/2008/v106n4a08.pdf

3. OMS: Organización Mundial de la Salud [Internet]. Región de las Américas: OMS; 2014. [Citado 06 Oct 2015]. Ceguera y discapacidad visual. o [aprox. 2 pantallas]. Disponible en: http:// www.who.int/mediacentre/factsheets/fs282/es/

4. Lafuente F, Alabarse C. Detección precoz de trastornos de la agudeza visual en escolares y su relación con el rendimiento escolar en 3er año del EGB1 de la Qocha. Facu Med [Internet]. 2007[citado 01 Oct 2015];8(1):16-20. Disponible en: http://www.fm.unt.edu.ar/ Dependencias/revistafacultad/vol_8_n_1_2007/cap3.pdf

5. Rincón IA, Rodríguez NC. Tamización de salud visual en población infantil prevención de la ambliopía. Repert Med Cir. [Internet]. 2009;[citado 2015 Mayo 17]18(4):210-217. Disponible en: https:// revistas.fucsalud.edu.co/index.php/repertorio/article/view/556/596

6. Molina MNP. Comparación de la efectividad de los optotipos Snellen y Bailey Lovie para medir la agudeza visual en pacientes entre cinco y doce años con ambliopía refractiva y ambliopía estrábica. Ciencia \& Tecnología para la Salud Visual y Ocular. 2008; [citado 2015 Mayo 17];10:19-28. Disponible en: https://ciencia.lasalle.edu. $\mathrm{co} / \mathrm{cgi} /$ viewcontent.cgi?article $=1111 \&$ context=svo

7. Deteccion oprtuna de alteraciones visuales en el prescolar en el primer nivel de atencion. Guía Práctica Clínica. Disponible: http:// www.imss.gob.mx/sites/all/statics/guiasclinicas/230GER.pdf

8. Capetillo BO, Triana CI, Martínez LZ, Roche CS. Broche HA. Frecuencia de la ambliopía en escolares. ScieloPediatr (Cuba). 2011; 83(4):372-381. Disponible en: http://scielo.sld.cu/scielo.php? script=sci_arttext\&pid=S0034-75312011000400005\#: :text=Al\% 201\%2C39\%20\%25\%20de\%20los, $7 \% 20$ a \%C3\%B1 os\%20 (tabla\% 203).

9. Rodríguez FA. Integración escolar de alumnos con deficiencia visual en España: algunas sugerencias espaciales y contribuciones tecnológicas y tiflotecnológicas. Estud Pedagóg. [Internet]. 2003;[citado 2015 may 17];29:143-153. Disponible en: http://www.scielo.cl/scielo. php?script=sci_arttext\&pid=S0718-07052003000100010

10. Rincon IA, Rodriguez NC, Tamización de salud visual en población infantil: prevención de la ambliopia. Articulo de investigacion cientifica y tecnologica. Disponible en: https://www.fucsalud.edu. co/sites/default/files/2017-01/8_2.pdf

11. Murillo FJ. La investigación sobre e icacia escolar a debate. Análisis de las críticas y aportaciones. Tendencias Pedagógicas. 2013;9:111-131. Disponible en: https://revistas.uam.es/ tendenciaspedagogicas/article/view/1842/1951

12. Rothenbach R, Carrillo M, Carrillo R, Rivera J. Ambliopía causada por errores refractarios. Rev Med HVQ. 2008; (12): 38-41. Disponible en: http://repositorio.puce.edu.ec/bitstream/ handle/22000/9869/TESIS\%20AGUDEZA\%20VISUAL\% 20DISMINUIDA\%20Y\%20RENDIMIENTO\%20ESCOLAR\% 20BAJO-\%20ELEANA\%20DEL\%20POZO-\%20VIVIANA\% 20LOPEZ.pdf?sequence $=1$

13. V Simposio Jornada de Investigación, Universidad Winer. Disponible en: https://xdoc.mx/preview/v-simposio-2014universidad-privada-norbert-wiener-5e58243eeb87c 